\title{
The Acute Effects of a Weight Loss Supplement on Endurance, Heart Rate, and Respiratory Exchange Ratio
}

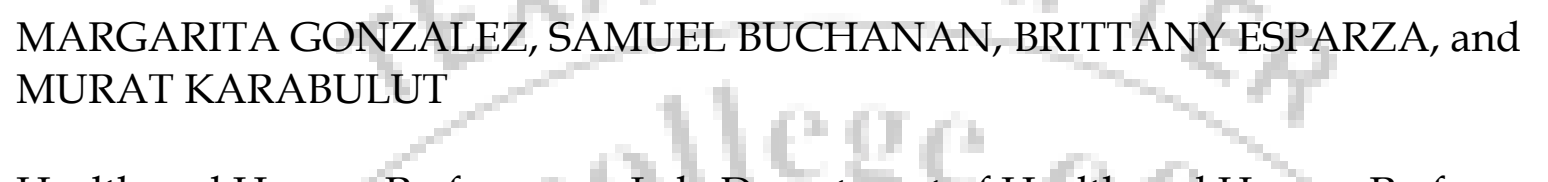

Health and Human Performance Lab; Department of Health and Human Performance; University of Texas Rio Grande Valley; Brownsville, TX

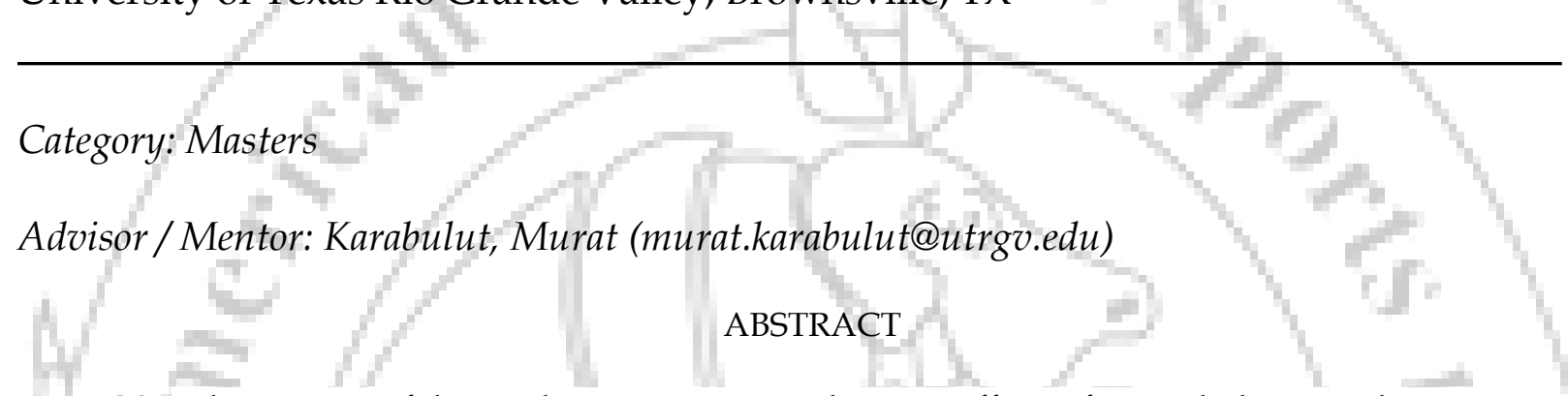

PURPOSE: The purpose of this study was to examine the acute effects of a weight loss supplement on endurance, heart rate (HR), and respiratory exchange ratio (RER). METHODS: Thirty-one (athletic and non-athletic) male and female subjects (male $=15$, female $=16$ ), between the ages of 18 and 40, were familiarized with the study protocol and performed the Bruce protocol on the first day. The study utilized a randomized, double blind, crossover, in which a subject would be given a placebo or supplement on two separate days. Each condition began with the subject reaching a hydration status at or below 1.010. Once the hydration levels were reached, the subject would receive an envelope, containing either supplement or placebo, to open and ingest 3 capsules. 30 minutes after the subject ingested the capsules, they would run on a treadmill, at $80 \% \mathrm{VO} 2 \mathrm{Max}$, until volitional fatigue. HR and RER were collected and monitored every three minutes during the running session. When the subject reached volitional fatigue, running time was recorded. RESULTS: There were no significant condition*time and condition*category interactions, but there were significant condition $(p=.03)$ and time $(p=.01)$ main effects for RER. There were also significant condition $(p=.03)$ main effects on the endurance for non-athletic females. Significant condition*ategory interaction $(\mathrm{p}<.01)$ and time main effects $(\mathrm{p}<.01)$ were observed for HR. CONCLUSIONS: The results showed that the weight loss supplement caused a significantly higher RER during the first 15 minutes of exercise. This may have been caused by the ingestion of caffeine resulting in vasoconstriction of the blood vessels surrounding the adipose tissue, causing a decrease in fatty acid mobilization, and therefore eliciting an increase in glycogen use. The non-athletic females may have had a greater response, which could be due to the greater caffeine to body weight ratio when compared to males and a lower daily caffeine ingestion than their athletic counterparts.

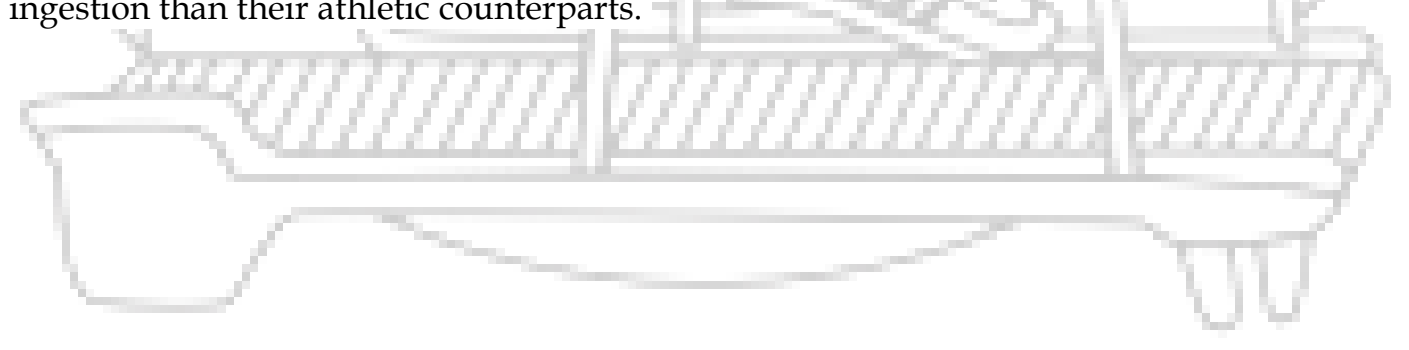




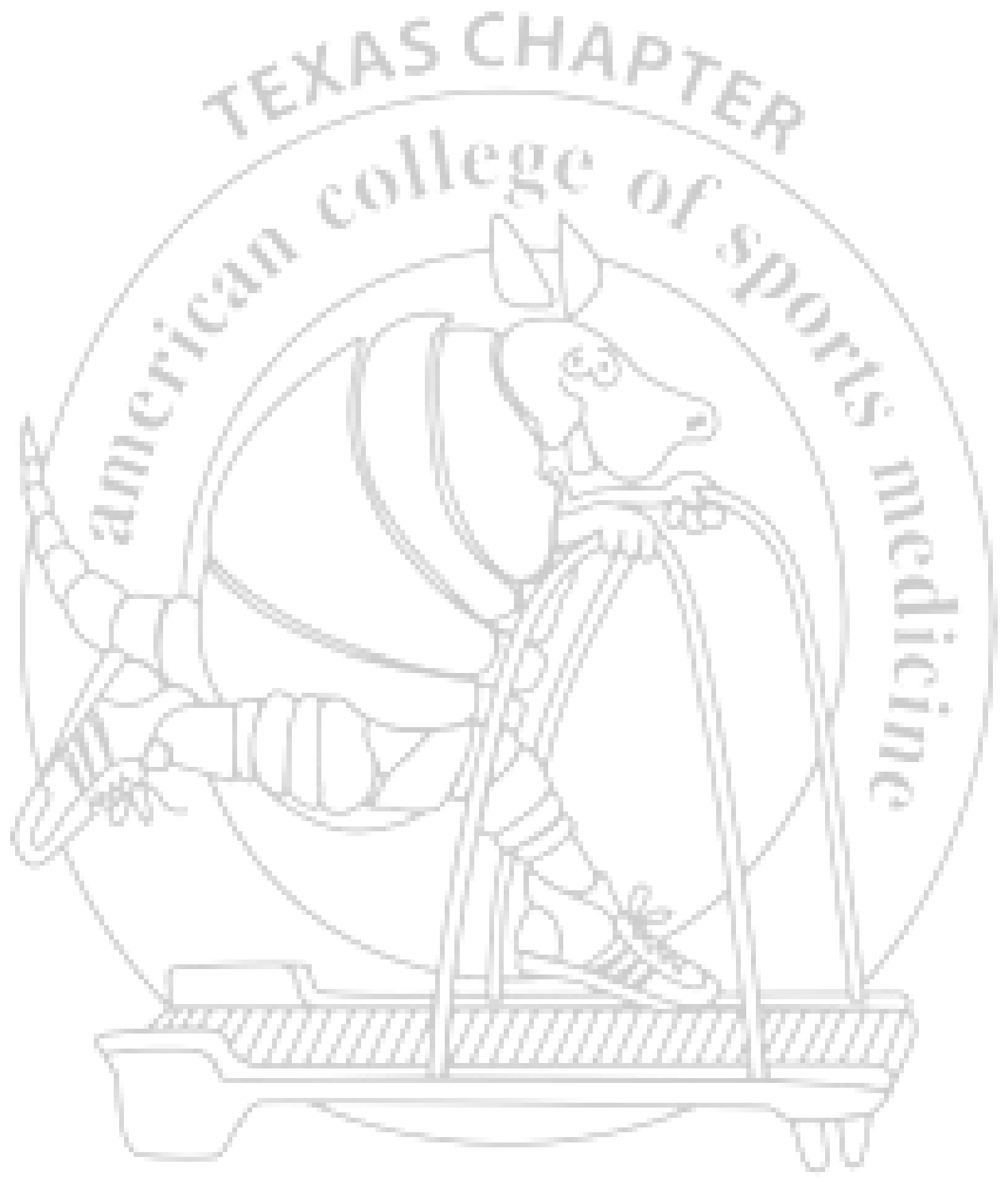

International Journal of Exercise Science

wWw.tacsm.org 